\title{
QOS MANAGEMENT IN MANUFACTURING GRID
}

Haiyang Sun, Tao Yu, Lilan Liu, Yu'an $\mathrm{He}$

CIMS \& Robot Center of Shanghai University, Shanghai University, China; Email: sunhaivang@263.net.

Abstract: In order to apply Manufacturing Grid technology to manufacturing field and provide resource-sharing and collaborating for the distributed manufacturing companies on a common platform, Manufacturing Grid system should resolve the problem brought by non-technical factors in manufacturing. Extending the requirement of product quality and regarding it as a kind of provided service which is required in the process of manufacturing. Then importing the idea of QoS (Quality of Service) to Manufacturing Grid and merging it into the manufacturing's characteristics. Based on it, making QoS management as the key research contents of Manufacturing Grid and create Manufacturing GridQoS (MG-QoS) system. Furthermore, proposing a kind of preliminary solution based on QoS management to Manufacturing Grid's practical application. It provides quality guarantee for the application of Manufacturing Grid and resolves the issue of prerequisite for Manufacturing Grid's practice from theoretical research.

Key words: Manufacturing Grid, Quality of Service (QoS), QoS Management

\section{INTRODUCTION}

The term "the Grid" was coined in the mid 1990s ${ }^{[1]}$, and the nature of the Grid is to share the distributed resources. The main aim of the Grid's application is to improve the resources' utilization ratio, reduce cost and complete nontrivial tasks those can't be finished without the Grid ${ }^{[2]}$.

In fact, resources are often leisure in manufacture enterprises because the utilization rations of the resources are low. On the contrary, many enterprises must collaborate with other enterprises because the resources of their own are limited.

This paper is supported by "SEC E-Institute: Shanghai High Institutions Grid" project.

Please use the following format when citing this chapter:

Sun, Haiyang, Yu, Tao, Liu, Lilan, He, Yu'an, 2006, in International Federation for Information Processing (IFIP), Volume 207, Knowledge Enterprise: Intelligent Strategies In Product Design, Manufacturing, and Management, eds. K. Wang, Kovacs G., Wozny M., Fang M., (Boston: Springer), pp. 831-839. 
There is a contradiction and it becomes more and more obvious for scarcities of effective collaboration, implementing and management. With the development of information process in manufacturing, people want to change this. Its keys are sharing resources and collaboration. In manufacturing field, cooperation frequently takes place in one enterprise or among them, and it can be regarded as various services. Furthermore, we extend the quality control in the manufacturing enterprise and build mechanism that can be used to evaluate the service provided by enterprise, and then establish Manufacturing Grid (MG) ${ }^{[3]}$. The Grid's core is Quality of Service (QoS) and its framework is Open Grid Service Architecture $(\mathrm{OGSA})^{[4]}$.

The application of Manufacturing Grid not only introduces the Grid technology into manufacturing, but also to promote the development of manufacturing and accelerate information process in manufacturing. Manufacturing Grid can achieve the aim of manufacturing to share distributed resources and realize coordinated manufacturing by Virtual Organization (VO) ${ }^{[s]}$.

This paper is organized as follows. In section 2, we import the conception of QoS into Manufacturing Grid, and then introduce Manufacturing GridQuality of Service (MG-QoS) and QoS management in Manufacturing Grid's application. In section 3, we analyze manufacturing resource management, task disassembling and manufacturing resource allocation. In section 4, we introduce Service Level Agreement (SLA) based on QoS and SLA management. In section 5, we summarize our research and conclude the paper.

\section{THE RESEARCH CONTENTS OF QOS IN MANUFACTURING GRID}

\subsection{QoS management in Manufacturing Grid}

The conception of Quality of Service was coined in information field. It refers to the comprehensive result of the service performance which decides the user's satisfaction. That is to say, QoS is a quality agreement about information transmission between the Service User (namely User) and Service Provider ${ }^{[6]}$, and between the clients and the integrated service network used to transfer information. However, Manufacturing Grid, as a new application of grid technology in manufacturing, provides a standard expandable network information platform by Grid technology and implements manufacturing resource's sharing and collaborating. 
Manufacturing Grid contains both characteristics of general grid and some unique differences from general computing grid. As an application of service grid in manufacturing, MG has some remarkable differences compared with other Grids, especially in resources, task and task's requirement. We can't import research of QoS from information field into manufacturing field directly. And in manufacturing, quality control impenetrate the whole process of manufacturing and contains almost requirements of products and reference points. So, we regard Manufacturing Grid-Quality of Service as the core of Manufacturing Grid platform system, and emphasize MG-QoS application management based on manufacturing process's characteristics ${ }^{[7]}$.

\subsection{Manufacturing Grid-Quality of Service}

We regard MG-QoS management as the core of Manufacturing Grid system. According to the ideas of Grid, we can say MG-QoS management is in the application layer of QoS management in manufacturing field. Its primary task is to deal with the logical affairs between user and service providers, and the interaction among different function modules. Just as illustrated in Figure.1.

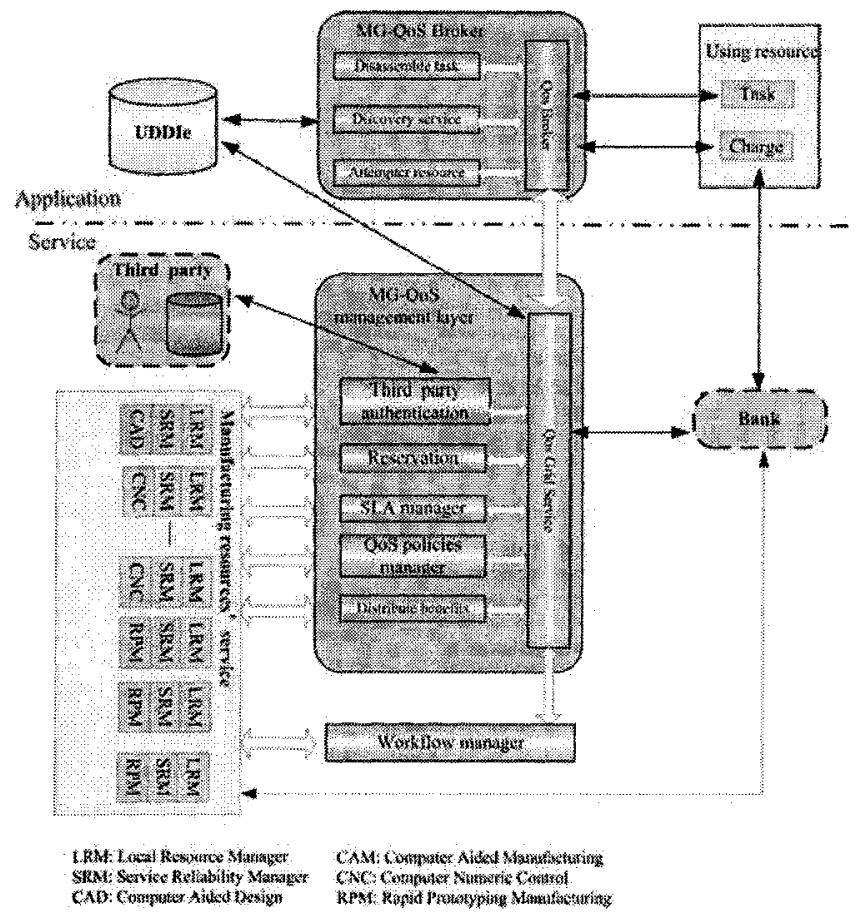

Figure 1. MG-QoS Architecture 


\subsection{QoS management in Manufacturing Grid application}

Then we introduce how to submit a task, and organize manufacturing, then finish task. From the whole process, we show how QoS management works.

When user has enrolled in Manufacturing Grid platform, he can submit task to platform ${ }^{[8]}$. The description and requirement of task are the contents of MG-QoS management. The manufacturing process in Manufacturing Grid likes the real process in manufacturing enterprise. Then, Manufacturing Grid system analyzes the task submitted by user and judge if the platform can complete user's task by the registered resources in platform. If platform ensures it can complete the user's task, then it disassemble user's task and contract with user. According to user's requirement of QoS and result of disassembling, Manufacturing Grid platform chooses optimal resource from all the registered resources to each subtask by some arithmetic. Following, the platform negotiate the details of subtask with resources' providers. If the provider accepts the subtask, then he can sign SLA agreement with the Manufacturing Grid platform. After signing, workflow management organizes manufacturing and executes workflow according to allocation of subtasks and requirements of QoS until the task is finished, and sends feedback to QoS management. While workflow management works, it can monitor the violation that happens in manufacturing process. If violation happens, workflow management will inform QoS management, and QoS management can executes punishment.

Following, we will introduce two modules which have close connections with QoS management.

\section{MANUFACTURING RESOURCES MANAGEMENT, TASKS DISASSEMBLING AND MANUFACTURING RESOURCES ALLOCATION}

\subsection{Establishing model of resource's QoS properties in Manufacturing Grid}

The resources in manufacturing field are different to those in information sciences, and furthermore, different manufacturing resource has different characteristics. So, according to these different characteristics, how to feasibly describe these characteristics' differences is the key to establish models of manufacturing resources' QoS properties ${ }^{[9]}$. 
First, we classify the resources of Manufacturing Grid as follows.

1) Technology resource

Technology is a kind of material methods for carrying out yielding, and it manifests the characteristic of manufacturing. It includes design resource, emulation resource, manufacturing resource and data exchange resource.

2) Information resource

Information resources include various manufacturing information. The resources provide necessary condition for managing and utilizing the manufacturing resources. Market information, management information, sharing information and cooperation information compose information resources.

3) Personnel resource

People as organizers or executants participate in almost all manufacturing process, so they play very important roles in the process. Personnel resources include manager, technical adviser, producer and service people.

4) Service resource

Service resources mainly include after service and training.

From the classifying of manufacturing resources, we can see that the manufacturing resources are heterogeneous, distributed, complicated and multifarious and their characteristics show the differences of QoS properties. So, when we establish the model of QoS properties, we must consider these properties' characteristics. Furthermore, in order to easily encapsulate the resources, build up resource nodes, manage resources and allocate resources, we should describe and define the resources' dynamic properties and static properties.

\subsection{Describe the user's task based on QoS}

If the user wants to take use of the convenience provided by Manufacturing Grid platform, first of all, he should submit his task to the platform. The platform will provide means for user to describe his task and the requirements of QoS. And we regard the requirement of product's quality and additive demands as the services that Manufacturing Grid platform should provide. Namely, user requires the platform to provide satisfying services and ensure good qualities. We introduce several dominating parameters to describe the task. The parameters include delivery time (T), product quality $(\mathrm{Q})$, manufacturing cost $(\mathrm{C})$ and service level (S). 


\subsection{Disassembling task and allocating resources based on QoS}

When user submitted task on the platform, the platform should analyze the whole task and disassemble it into subtasks, and then ensure if it can complete the user's task. The platform's analyzing includes two steps. Firstly, the platform adopts different Global Process Planning (GPP) template to disassemble the task simply. Secondly, the expert belonged to Manufacturing Grid platform will check up the disassembling result.

When the platform decides to accept the task based on the disassembling result, the expert will perfect the result, and synchronously, he should decide the manufacturing method and process. Then, he will rake all the resources they are capable of completing one subtask, and choose the optimal resource. We adopt same method to describe the resources, and then we can distinguish the different resources. We also introduce several dominating parameters to describe the different resources in detail. And the parameters also include delivery time $(\mathrm{T})$, product quality $(\mathrm{Q})$, manufacturing $\operatorname{cost}(\mathrm{C})$ and service level(S) $\left.{ }^{[10},{ }^{11}\right]$. The description of resource is similar to the description of task, so we can easily rake resources and choose optimal one.

\section{ERVICE LEVEL AGREEMENT (SLA) MANAGEMENT}

\subsection{General SLA management and Manufacturing Grid's SLA management}

In information business, using information resource is a series of business activities. In order to ensure the rights and the obligations of the user and the provider, the two parties will establish agreements. SLA (Service Level Agreement) is the exact statement of all expectations and obligations in the business relationship between the provider and the user. It is built on the agreement formed by contract between user and service provider, and its purpose is guaranteeing the interest of both parties. In information field, SLA management includes a kind of regular document and can automatically collect useful information and evaluate the parameters in SLA agreement.

Because of the characteristics of manufacturing, SLA management in Manufacturing Grid is different from the SLA management in other fields. The main research of SLA includes negotiation among user, provider and the 
platform, signing SLA and conserving SLA. Furthermore, the SLA management can provide proof for violation of SLA, analyze breach and execute punishment.

\subsection{SLA management's research and its framework in Manufacturing Grid}

The main research of SLA management includes the following.

1) Designing SLA management module and interfaces;

2) Establishing SLA contract repository and creating multifarious regulations;

3) Monitoring violation, such as communicating with workflow management, analyzing violation's reason, resuming the interrupted task and recording the violation information;

4) Executing punishment, including deciding the punishment's grade and renewing punishment contents in punished resource's property;

5) Conserving the signed SLA documents.

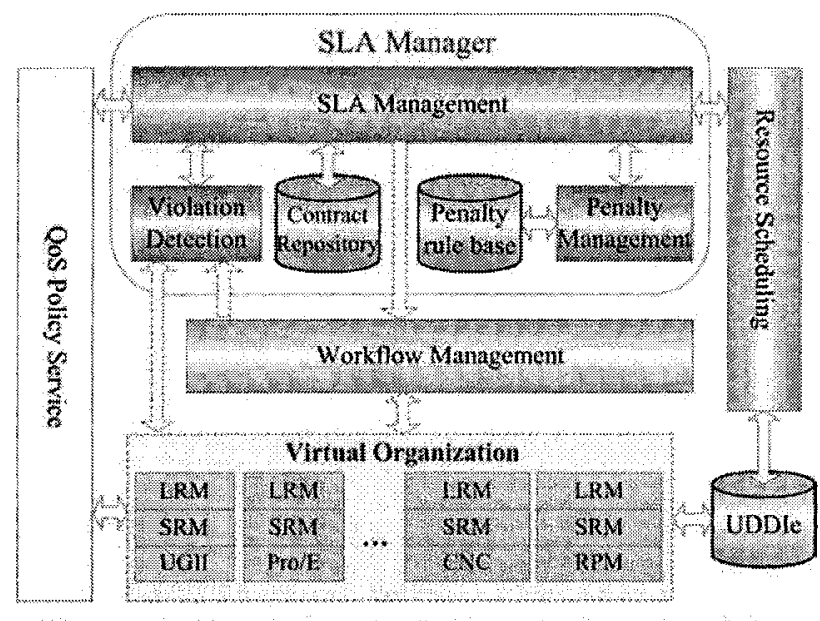

CNC Computer Numeric Control RPM: Rapid Prototyping Manufacturing

Figure 2. SLA manager

Figure.2 illustrates the framework of SLA Management module. From Figure.2, we can see SLA management module mainly includes SLA Management, Violation Detection, Contract Repository, Penalty Management, Penalty Rule Base and QoS Policy. 


\subsection{SLA management}

SLA management is the dominating content of SLA management module, and it can interact with other functions of the module and manage the module. The main of SLA management includes following.

1) User negotiates with platform about QoS; Platform negotiates with provider about QoS;

2) Create SLA document;

3) Submit workflow to workflow management;

4) When Violation Detection finds a case happens, it will evaluate the violation and decide the punishment, and then penalty management will execute punishment;

5) SLA Management rectifies or resumes the allocation of resources based on the different violations in order to complete the task;

6) Terminate SLA dialogue and renew properties of resources which have provided service.

\subsection{SLA template}

Because of complexity of manufacturing resources and diversity of functions, the descriptions of their services are different. In order to simplify the platform's management, we design different templates to different services.

SLA templates provide criterion for SLA document. If create a SLA agreement, first, people choose a template from the template repository according the service of the provider. Second, people make sure the requirement of this SLA. Then, finish the details of SLA and create relevant document.

\subsection{SLA executing}

When finish SLA's creating, SLA management will submit formal workflow to workflow management and the management will execute and manage the whole workflow. It includes informing provider's obligation, monitoring violation and starting punishment when violation happens. The whole process should run until SLA finish.

\section{CONCLUSION}

Grids are rapidly emerging as a practical means by which to perform new science and new applications. Manufacturing Grid helps the enterprises with 
similar goals and interests form loosely coupled virtual organizations for collaboration and resource sharing as one of grid applications. In this paper we mainly discuss the QoS management. We extend the requirements of product's quality to the requirements of service provided by provider, and bring forward a method of Manufacturing Grid's application based on QoS management. We introduce the process of Manufacturing Grid's application in detail, and propose the appropriate method to solving practical issues. The implementation in the project of Manufacturing Grid Platform supported by "SEC E-Institute: Shanghai High Institutions Grid" shows that the research is valuable and the solution is effective.

\section{REFERENCES}

1. Global Grid Forum, http://www.gridforum.org.

2. L. Pearlman, V. Welch, I. Foster, and C. Kesselman,"A Community Authorization Service for Group Collaboration", Proceedings of the IEEE 3rd International

3. LIU Lilan, YU Tao, SHI Zhanbei. Research of Self-Organization Manufacturing Grid and its Task Scheduling Algorithm . Computer integrated manufacturing system, 2003, 9 (6),pp.449-455

4. Foster, C. Kesselman, J. Nick, S. Tuecke , "The Physiology of the Grid: An Open Grid Services Architecture for Distributed Systems Integration," Open Grid Service Infrastructure $W G$, Global Grid Forum, June 22, 2002.

5. Ian Foster, Carl Kesselman, Steven Tuecke. The Anatomy of the Grid: Enabling Scalable Virtual Organizations . Intl J.Supercomputer Applications, 2001, 15 (3),pp.1-21.

6. I.Foster, C.Kesselman, J.M.Nick and S. Tuecke . Grid Service for Distributed System Integratiopn .IEEE Computer, 2002, 35 (6),pp.37-46.

7. SHI Zhanbei. Research of OGSA-based Manufacturing Grid QoS System . Shanghai: Shanghai University, 2004.

8. Shi Zhanbei, Yu Tao, Liu Lilan, "Service Registry and Discovery in Rapid Manufacturing Grid", Computer Applications, 2003, pp.85-87

9. Liu Lilan, Yu Tao, Shi Zhanbei, "Research on Rapid Manufacturing Grid and Its Service Nodes", Machine Design and Research, 2003, pp.57-59.

10. ZHAO Nai-yan, FAN Yu-shun. A Product-Structure-Based Solution to Select Collaborating Partners of Agile Virtual Enterprise, Computer Integrated Manufacturing Systems, 2002, 8 (2),pp.99-104. 